\title{
Video Article \\ Bioenergetics and the Oxidative Burst: Protocols for the Isolation and Evaluation of Human Leukocytes and Platelets
}

\author{
Philip A. Kramer* ${ }^{1}$, Balu K. Chacko* ${ }^{* 1}$, Saranya Ravi ${ }^{1}$, Michelle S. Johnson ${ }^{1}$, Tanecia Mitchell ${ }^{1}$, Victor M. Darley-Usmar* \\ ${ }^{1}$ UAB Mitochondrial Medicine Laboratory, Center for Free Radical Biology, Department of Pathology, University of Alabama at Birmingham \\ * These authors contributed equally
}

Correspondence to: Victor M. Darley-Usmar at darley@uab.edu

URL: https://www.jove.com/video/51301

DOI: doi:10.3791/51301

Keywords: Immunology, Issue 85, bioenergetics, translational, mitochondria, oxidative stress, reserve capacity, leukocytes

Date Published: $3 / 27 / 2014$

Citation: Kramer, P.A., Chacko, B.K., Ravi, S., Johnson, M.S., Mitchell, T., Darley-Usmar, V.M. Bioenergetics and the Oxidative Burst: Protocols for the Isolation and Evaluation of Human Leukocytes and Platelets. J. Vis. Exp. (85), e51301, doi:10.3791/51301 (2014).

\section{Abstract}

Mitochondrial dysfunction is known to play a significant role in a number of pathological conditions such as atherosclerosis, diabetes, septic shock, and neurodegenerative diseases but assessing changes in bioenergetic function in patients is challenging. Although diseases such as diabetes or atherosclerosis present clinically with specific organ impairment, the systemic components of the pathology, such as hyperglycemia or inflammation, can alter bioenergetic function in circulating leukocytes or platelets. This concept has been recognized for some time but its widespread application has been constrained by the large number of primary cells needed for bioenergetic analysis. This technical limitation has been overcome by combining the specificity of the magnetic bead isolation techniques, cell adhesion techniques, which allow cells to be attached without activation to microplates, and the sensitivity of new technologies designed for high throughput microplate respirometry. An example of this equipment is the extracellular flux analyzer. Such instrumentation typically uses oxygen and $\mathrm{pH}$ sensitive probes to measure rates of change in these parameters in adherent cells, which can then be related to metabolism. Here we detail the methods for the isolation and plating of monocytes, lymphocytes, neutrophils and platelets, without activation, from human blood and the analysis of mitochondrial bioenergetic function in these cells. In addition, we demonstrate how the oxidative burst in monocytes and neutrophils can also be measured in the same samples. Since these methods use only 8-20 ml human blood they have potential for monitoring reactive oxygen species generation and bioenergetics in a clinical setting.

\section{Video Link}

The video component of this article can be found at https://www.jove.com/video/51301/

Introduction

Monitoring the bioenergetic health of immune cells (monocytes, lymphocytes, neutrophils) and platelets from blood has been recognized for some time as a potentially useful diagnostic tool to assess the overall bioenergetic health of an individual. There is an emerging body of literature attributing a number of diseases such as cancer, cardiovascular diseases and neurodegenerative diseases to mitochondrial dysfunction ${ }^{1,2}$. This is clinically important since mitochondrial dysfunction can initiate a series of cellular events that promote pro-inflammatory signaling pathways or lead to cell death. Several studies have characterized mitochondrial function of peripheral blood mononuclear cells and platelets in conditions such as fibromyalgia, diabetes, septic shock, and Alzheimer's disease $e^{3-7}$. For example, a recent study evaluated the bioenergetics of platelets as a marker for mitochondrial function and found that platelets from Type 2 diabetic patients had diminished mitochondrial oxygen consumption ${ }^{7,8}$. From these findings and others, it is clear that monocytes, lymphocytes, neutrophils, and platelets can serve as surrogate markers of changes in bioenergetics under pathological conditions since they survey the systemic circulation and may reflect local and global metabolic changes. To determine whether this approach has prognostic or diagnostic value a high throughput method of analysis and a consistent method for cell preparation is required.

The methods to measure mitochondrial function in leukocytes and platelets have previously involved isolation of mitochondria or assessment of cellular bioenergetics in intact cells ${ }^{4,9}$. The advantage of cellular bioenergetic assessment using an extracellular flux (XF) analyzer is that the mitochondrial function in cells can be established with endogenous substrates and respiratory parameters such as proton leak and maximal respiratory capacity can be determined. We and others have used this technology to show that bioenergetic profiles in platelets, monocytes and lymphocytes isolated from human blood can be established and compared among cell types ${ }^{8}$. In addition, both neutrophils and monocytes possess an oxidative burst capacity in which NAPDH oxidases are activated and consume oxygen to form superoxide. Importantly, this pathway is a key component of innate immunity and is modulated by systemic inflammation. For example, it has been shown that changes in the oxidative burst capacity are associated with various autoimmune diseases such as multiple sclerosis, arthritis, and recurrent infections ${ }^{10,11}$. Currently there are no high throughput quantitative assays available to measure the oxidative burst in clinical samples. This is important since characterizing the oxidative burst capacity of neutrophils and monocytes may also serve as an important diagnostic tool for several pathologies.

The key technical challenges have been low sensitivity for measurement of oxygen consumption using conventional polarographic techniques and the need to use attached cells when using more sensitive microplate fluorometric techniques. In this practical video, we describe the 
technical solution to these problems. We detail the methods for the isolation, plating and measurement of bioenergetics of monocytes, lymphocytes, neutrophils, and platelets and the oxidative burst of monocytes and neutrophils from human blood. This method is suitable for clinical assessment of bioenergetics and oxidative burst for investigators that have access to a patient population and the capability to obtain fresh blood samples.

Protocol

All protocols described in this manuscript for blood collection, isolation and analysis have been reviewed and approved by the Institutional

Review Board at the University of Alabama at Birmingham.

\section{Cell Isolation (Modified from Histopaque Sigma-Aldrich Protocol No. 1119 and Miltenyi Biotec MicroBead Positive and Negative Selection Protocols)}

1. Centrifuge whole blood (collected in ACD or EDTA tubes) at $500 \mathrm{xg}$ for $15 \mathrm{~min}$ in a centrifuge with a swinging-bucket rotor (acceleration $=$ $5-6$, and no brake).

2. Remove the top layer that contains the platelet rich plasma (PRP) with a transfer pipette until $1 \mathrm{~cm}$ remains above the cell layer (erythrocyte/ buffy coat). Set aside the PRP at room temperature for processing later.

3. Transfer the buffy coat to a sterile $50 \mathrm{ml}$ conical tube, dilute to $24 \mathrm{ml}$ with basal RPMI or to at least $4 \mathrm{x}$ the starting buffy coat volume. Note: The upper half of the RBC layer may have to be included although this will result in increasing the RPMI volume proportionally.

4. Prepare the density gradient using low density Ficoll with a specific gravity of 1.077 (1077) for the top layer and high density Ficoll with a specific gravity of 1.119 (1119) for the bottom layer in three $15 \mathrm{ml}$ conical tubes ( $3 \mathrm{ml}$ each). To perform this step, first add 1077 to each tube. Using a $5 \mathrm{ml}$ narrow pipette add 1119 to the bottom of the tube by placing the pipette tip beneath 1077 and slowly release the reagent without mixing with the upper gradient. A total of $6 \mathrm{ml}$ of density gradient media should be present at this stage with a visible phase of separation at the $3 \mathrm{ml}$ mark.

5. Using an automated pipette, add $8 \mathrm{ml}$ of diluted blood (from step 1.3) gently to each gradient tube using the low-power setting to prevent from disturbing the gradient layers. The total volume should be $14 \mathrm{ml}$ at this step.

6. Centrifuge tubes at $700 \times \mathrm{g}$ for $30 \mathrm{~min}$ at room temperature (acceleration 6 , no brake) Note: At this stage, three distinct cell bands should be evident (Figure 1A). The uppermost bands (between 1077 and plasma) contains mononuclear cells (MNCs) and platelets, and the middle band (between 1007 and 1119) contains polymorphonuclear cells (PMNs) and lower band (below 1119) contains RBCs.

7. Collect the MNC and PMN separately by using sterile glass pipettes without disturbing the other cell bands. Combine the MNC population from each tube into a sterile $50 \mathrm{ml}$ conical tube. Repeat this for the PMN population as well.

8. Add 4 volumes of RPMI to each tube containing the MNC and PMN fractions respectively to dilute the density gradient.

9. Centrifuge tubes at $700 \times \mathrm{g}$ for $10 \mathrm{~min}$ at room temperature.

10. Resuspend MNC cell pellet and PMN cell pellet in $1 \mathrm{ml}$ RPMI containing $0.5 \%$ ultra-pure fatty acid-free BSA (RPMI-BSA) and transfer to sterile $1.5 \mathrm{ml}$ tubes. Pellet the cells using a benchtop picofuge for $30 \mathrm{sec}$.

11. Discard the supernatant and resuspend each cell pellet in $80 \mu \mathrm{IPMI}-\mathrm{BSA}$.

12. Add $20 \mu \mathrm{l}$ of magnetic bead labeled-antiCD14 antibody to the tube containing the MNC fraction for positive selection of monocytes. For the tube containing the PMN cell fraction, add $20 \mu$ l of magnetic bead labeled-antiCD15 antibody for positive selection of neutrophils. Incubate each cell suspension for $15 \min$ at $4^{\circ} \mathrm{C}$.

13. Wash each cell suspension with $1 \mathrm{ml}$ RPMI-BSA media and pellet as before and resuspend each pellet in $500 \mu \mathrm{l}$ RPMI-BSA.

14. The labeled cells are separated in the magnetic field of the magnetic activated cell sorting (MACS) separator. Prepare the MACS LS columns (one for each cell suspension) by placing the column on the MACS separator and washing with $3 \mathrm{ml}$ of RPMI-BSA prior to adding the cell suspension.

15. After applying cell suspensions, wash each column $3 x$ with $3 \mathrm{ml}$ of RPMI-BSA media (make sure to let the media drain completely between washes). Collect the cell suspension flow-through and column washes into a sterile tube.

16. To isolate monocytes and neutrophils, remove the column from the magnetic field after the final wash and elute the positively selected cells into a sterile tube with $5 \mathrm{ml}$ of RPMI-BSA using the column plunger.

17. To isolate lymphocytes pellet the flow-through-wash fraction of the MNCs from step 1.15 at $300 \times \mathrm{g}$ for $10 \mathrm{~min}$. Discard the supernatant and resuspend the cell pellet in $80 \mu \mathrm{l}$ RPMI-BSA. Add $20 \mu \mathrm{l}$ of CD61 and CD235a antibodies and incubate cell suspension for 15 min at 4 ${ }^{\circ} \mathrm{C}$. Repeat the MACS separation as before and collect the flow though containing the lymphocytes.

18. To pellet monocytes, neutrophils, and lymphocytes fractions, centrifuge as before (step 1.9) and discard supernatants. Cell pellets should be resuspended in $1 \mathrm{ml}$ extracellular flux media (XF-DMEM) for counting.

Note: $8 \mathrm{ml}$ of whole blood should result in $1-5 \times 10^{6}$ monocytes $/ \mathrm{ml}$, and 5-20 × $10^{6}$ lymphocytes and neutrophils $/ \mathrm{ml}$.

19. To isolate platelets, centrifuge PRP (step 1.2) for $8-10 \mathrm{~min}$ at $1,500 \mathrm{xg}$ at room temperature. Remove the plasma and wash cell pellet once with sterile $5 \mathrm{ml}$ PBS supplemented with $1 \mu \mathrm{g} / \mathrm{ml} \mathrm{PGI}_{2}$, repellet and suspend final pellet in $1 \mathrm{ml}$ of PBS-PGI ${ }_{2}$ buffer.

20. Determine platelet count by turbidimetry once the platelets are suspended in $\mathrm{PBS}-\mathrm{PGI}_{2}$ buffer by spectrophotometer as described by Walkowiak et al. using the following equation: $\left[6.23 /\left(2.016-\mathrm{Abs}_{800}\right)\right]-3.09 \times$ dilution factor $=\# \times 10^{8}$ platelets $/ \mathrm{ml}^{12}$.

\section{Plating of the Cells}

1. Preparation of Cell-Tak (cell adhesive): Add $90 \mu \mathrm{l}$ cell adhesive to $180 \mu \mathrm{liH}_{2} 0,540 \mu \mathrm{l}$ of $0.1 \mathrm{~N}$ sodium bicarbonate ( $\mathrm{pH} 8.0$ ), and adjust $\mathrm{pH}$ to 7.2-7.8 using $1 \mathrm{~N} \mathrm{NaOH}$. Prepare XF plates (24-well, $\mathrm{V} 7$ microplates) by coating each well with $30 \mu \mathrm{l}$ of prepared cell adhesive.

Note: The cell adhesive should be prepared fresh for each use.

2. Incubate plate at $37^{\circ} \mathrm{C}$ for $20-30 \mathrm{~min}$ then aspirate cell adhesive and wash wells with PBS twice (1 ml/well).

3. Perform a cell count on isolated monocytes, lymphocytes, neutrophils and platelets and bring to appropriate volume using XF-DMEM to allow a seeding density of $250 \mathrm{k}$ cells/well for monocytes, lymphocytes, and neutrophils, and $25 \times 10^{6} /$ well for platelets. Alternatively, to measure 
oxidative burst response, neutrophils can be seeded at $125 \mathrm{k}$ cells/well. The final seeding volume for each cell suspension should be $200 \mu \mathrm{l} /$ well.

4. Centrifuge the plate at $200 \mathrm{xg}$ for $1 \mathrm{sec}$ with no brake. Rotate the plate $180^{\circ}$ and centrifuge again with no brake at $300 \mathrm{xg}$.

5. Bring final well volume to $660 \mu \mathrm{l}$ with XF-DMEM and incubate at $37^{\circ} \mathrm{C}$ for $30 \mathrm{~min}$ prior to XF assay. Note: Photomicrographs of plated cells before and after the assay can be used to ensure adequate plating and rule out cell detachment.

\section{Preparation of a 24-well Extracellular Flux Assay Plate (XF24)}

1. Hydrate XF probes with calibrant solution for a minimum of $2 \mathrm{hr}$ prior to assay.

2. Prepare $10 \mathrm{x}$ stocks of $0.5 \mu \mathrm{g} / \mathrm{ml}$ oligomycin, $0.6 \mu \mathrm{M} \mathrm{FCCP}$, and $10 \mu \mathrm{M}$ antimycin A in XF-DMEM and load $75 \mu$ l of stock solutions into cartridge injection ports in the above order. If oxidative burst measurements are to be obtained, injection of $10 x$ stock of $100 \mathrm{ng} / \mathrm{ml}$ PMA can be injected after antimycin-A.

3. Calibrate the hydrated and loaded cartridge and perform the XF assay. The detailed methods for analysis and interpretation of both oxygen consumption and $\mathrm{pH}$ changes have been described in previous publications $\mathrm{s}^{9,13,14}$ and are not discussed in detail here.

4. After XF assay, aspirate all but the last $50 \mu \mathrm{l}$ of XF-DMEM after assay to prevent loss of cells from the plate and add $50 \mu \mathrm{ll}$ RIPA cell lysis buffer and perform a protein assay for normalization.

\section{Representative Results}

In order to assess the bioenergetics of blood cells, whole blood is collected from human subjects by venipuncture in an EDTA or ACD vacutainer collection tube. The isolation of leukocytes and platelets following blood collection is visualized in Figure 1A as detailed in the protocol. Whole blood samples are processed within $8 \mathrm{hr}$ of collection to avoid cell death and activation. Extended storage times greater than $8 \mathrm{hr}$ have not been tested but may result in altered bioenergetics and be less representative of physiological conditions. Acid citrate dextrose (ACD-8 ml) and EDTA vacutainer tubes have been used for cell isolations with no observed effect on the bioenergetic function of isolated cells. Anticoagulants are necessary as clots reduce the number of cells obtained, interfere with proper phase formation during Ficoll gradient centrifugation, and result in little to no platelets obtained in centrifuged serum. All blood represents a biological hazard and proper personal protective equipment must be implemented throughout the entire procedure even after cell populations are isolated or cell lysates are generated. Samples are to be disposed of in accordance with the institution's human waste disposal standards.

Whole blood is centrifuged to separate the platelet rich plasma from the buffy coat, the white layer just above the packed red blood cells which contains the leukocytes. The buffy coat is applied to density gradients and centrifuged to obtain the peripheral blood mononuclear cells (monocytes and lymphocytes) and polymorphonuclear cells (granulocytes). RBC contamination of the individual MNC and PMN cell phases is common at this stage and should be resolved during the MACS purification. The various cell types are then obtained by incubation with magnetic antibodies to obtain pure fractions. Monocytes are collected by incubation of the PBMC fraction with CD14, the flow-through from the monocytes contain lymphocytes, which are then depleted of RBCs and platelets. The neutrophils are obtained by incubation of the polymorphonuclear cell layer with CD15 antibody (Figure 1A). Thereafter, the platelets can be pelleted by centrifugation of the platelet rich plasma and washed to remove other plasma components. After these selections the platelets, CD14+ monocytes, lymphocytes, CD15+ neutrophils can be counted and plated on a cell adhesion medium-coated XF analyzer plate for bioenergetics and oxidative burst analysis.

Each stage of the procedure should be performed in sterile conditions and is encouraged to prevent the premature activation of the oxidative burst response in monocytes and neutrophils. An indicator of less than sterile conditions during the isolation is evident when neutrophils, which have little to no oxygen consumption under basal conditions, begin the extracellular flux assay with elevated OCR measurements that gradually increase or decline throughout the assay. We stress the importance of imaging cells using light microscopy at 200X or greater to ensure that no morphological signs of activation have occurred prior to the assay. For example, neutrophils normally display a rounded to irregular shape with pronounced cell boundaries as seen in Figure 1B. However, these cells will flatten against the surface of the plate when activated and lose their distinct cell morphology. We have previously reported the morphological changes of activated leukocytes and platelets using this protocol and additional measures must be taken to ensure sterile conditions if encountering activation ${ }^{8}$.

A simplified scheme of the protocol is seen in Figure 2 as a time table in which the primary steps of the cell isolation, XF plate preparation, and XF assay are detailed. Isolation consists of the density gradient separation of cell types and is followed by magnetic separation. Parallel to the cell isolation process, XF plate preparation is necessary to avoid delays in cell plating or beginning the XF assay. This is critical because significant delays can lead to cell activation and diminished bioenergetics parameters.

Inadequate cell concentrations after isolation are a common problem and optimization of each centrifugation process is necessary to avoid cell loss. Successful isolations have been performed on as little as $6 \mathrm{ml}$ of blood but alterations can be made depending on circulating concentrations of the desired cell type. If the density gradient is improperly prepared, distinct cell phases might not be visible and loss of cells can occur (see JoVE instructional video for a visual demonstration). Insufficient platelet isolated from the PRP is rare but has occurred if washing buffer does not contain $\mathrm{PGI}_{2}$ or if the isolation takes place below room temperature. The risk of platelet activation is often prevented if platelets are the last cells to be isolated; however, if platelets remain in washing buffer for extended periods the bioenergetics will be affected. There have been instances of smaller platelet populations that did not pellet during the $1,500 \times \mathrm{g}$ centrifugation and the bioenergetics of these cells has not been extensively characterized. See Table 2 for a more complete troubleshooting guide.

The bioenergetic profiles of leukocytes and platelets can be determined using the XF analyzer, which measures real-time $\mathrm{O}_{2}$ consumption in cells noninvasively. Each cell type is plated on the XF24 plate with five replicates as shown in the Figure 3A. Table 1 indicates the expected basal and oxidative burst values by cell type during the assay and the average protein concentrations per well. Higher throughput technologies are available, such as the XF96, which allows for four donors to be assessed simultaneously as shown in Figure 3B. After the assay, the data is exported to a work-station computer for analysis using the appropriate XF software and Microsoft excel. The OCR values were normalized to total protein content in the corresponding wells and expressed as pmol/min/mg protein. The representative bioenergetic-oxidative burst profiles 
of each cell type were recently reported by our group ${ }^{8}$. Further analysis of bioenergetic parameters of the assay (basal, ATP-linked, proton leak, maximal, nonmitochondrial and oxidative burst) can be calculated for individual wells as previously shown and described below ${ }^{8}$.

The basal oxygen consumption rate (OCR) is established by the first 3 measurements as shown in Figure $4 \mathrm{~A}$. Oligomycin $(0.5 \mu \mathrm{g} / \mathrm{ml})$, an inhibitor of mitochondrial ATP-synthase is injected into the XF medium to estimate the OCR coupled to ATP synthesis and represented as ATPlinked. The residual OCR minus the nonmitochondrial OCR can be attributed to proton leak. Then FCCP an uncoupler is added to determine the maximal OCR, followed by antimycin-A, an inhibitor of mitochondrial respiration, to determine nonmitochondrial sources of oxygen consumption. Reserve capacity is a measure of the amount of ATP that can be produced under energetic demand and can be calculated as the difference between the maximum rate of respiration and the basal. In order to determine the oxidative burst capacity, phorbol 12-myristate 13-acetate (PMA) a PKC agonist is injected to increase NADPH oxidase activity, and the increase in oxygen consumption rate following PMA stimulation can be measured using the XF analyzer. Figure 4B show how to calculate the different parameters of mitochondrial function and oxidative burst.

A

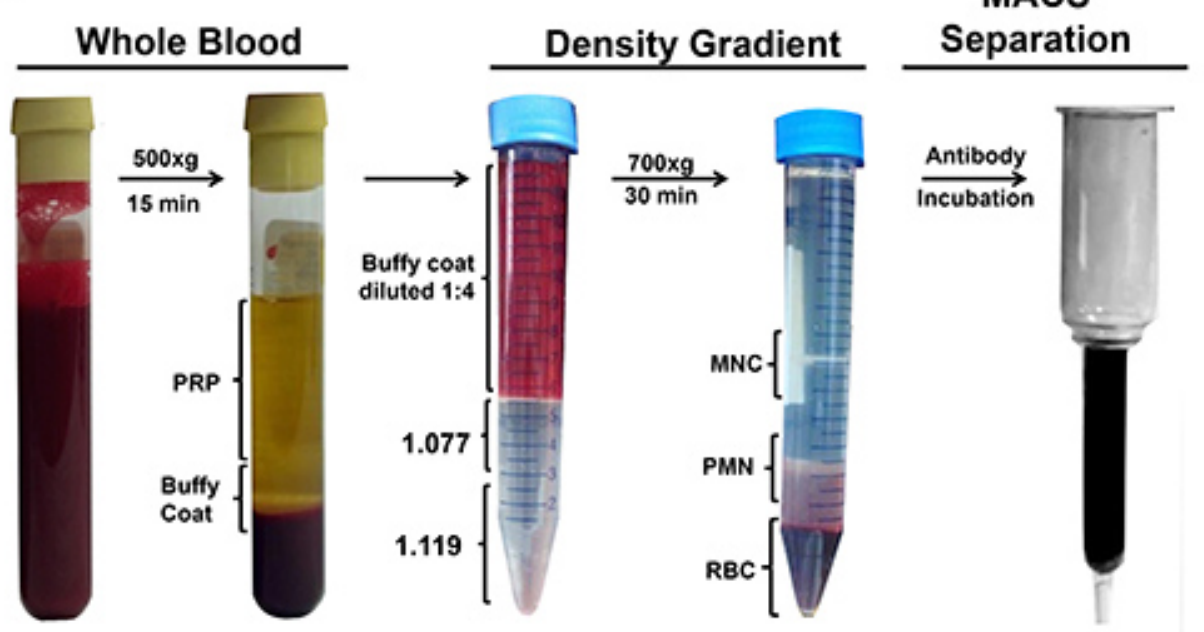

B
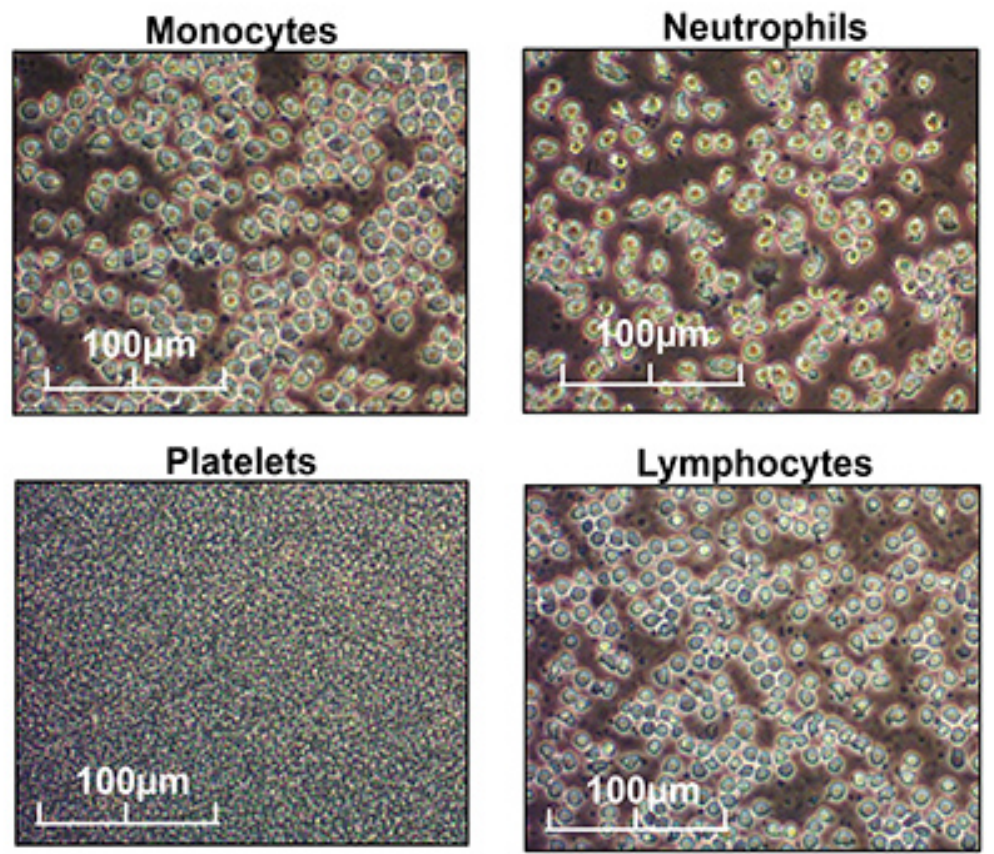

Figure 1. Isolation of Leukocytes and Platelets from Whole Blood. A) Freshly collected specimens are separated into their plasma and cellular components by centrifugation and purified by Ficoll density gradient, and Magnetic Activated Cell Sorting (MACS) by positive (monocyte and neutrophil) or negative selection (lymphocytes), while platelets are isolated by high-speed centrifugation of the platelet rich plasma. B) Images of the isolated cell populations once resuspended in XF DMEM and plated at indicated cell densities. Please click here to view a larger version of this figure. 


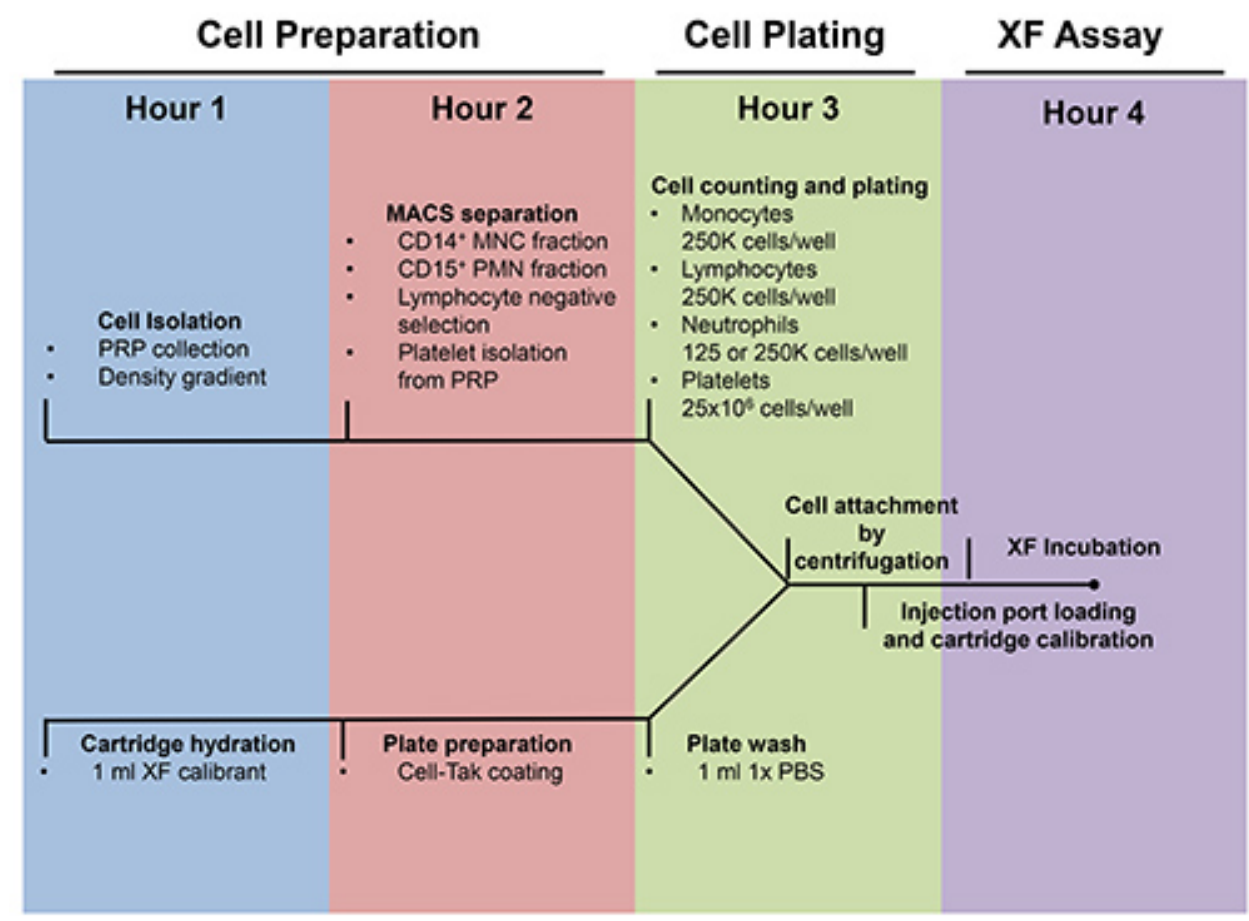

Figure 2. Preparation of XF24 plates for Bioenergetic studies of leukocytes and platelets. Detailed timeline for the preparation of a XF24 assay plate by cell isolation and plating and cartridge hydration and injection port loading. 
A

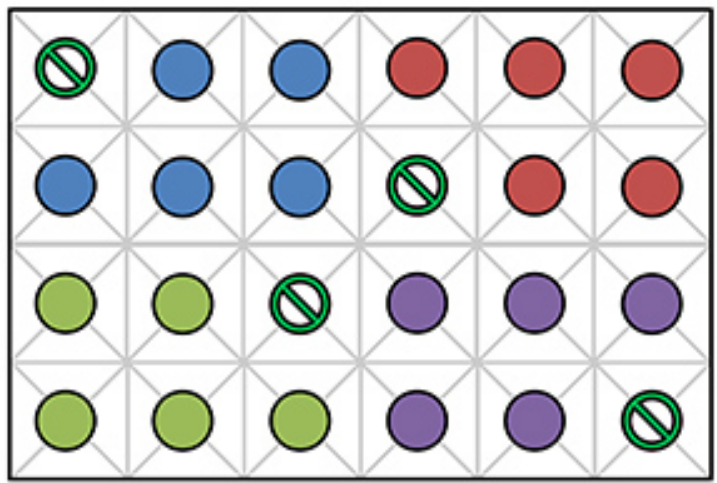

Plate setup:

Monocytes (250k / well)

Lymphocytes (250k / well)

Neutrophils (125-250k / well)

Platelets $\left(25 \times 10^{6} /\right.$ well $)$

Blank (Q)

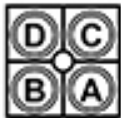

A: Oligomycin $(0.5 \mu \mathrm{g} / \mathrm{ml})$

B: $F C C P(0.6 \mu \mathrm{M})$

C: Antimycin A $(10 \mu \mathrm{M})$

D: PMA (100 $\mathrm{ng} / \mathrm{ml})$

B

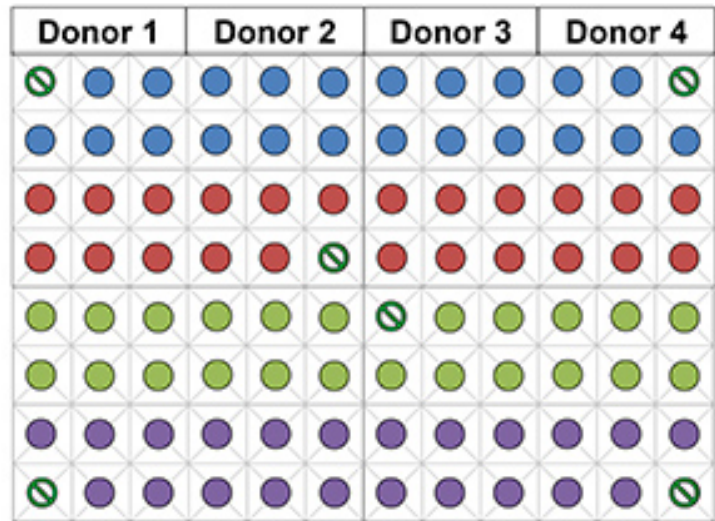

\section{Plate setup:}

Monocytes (150k / well)

Lymphocytes (150k / well)

Neutrophils (75-150k / well)

Platelets $\left(10 \times 10^{6} /\right.$ well)

Blank $\boldsymbol{Q}$

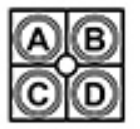
A: Oligomycin $(1 \mu \mathrm{g} / \mathrm{ml})$
B: $\operatorname{FCCP}(0.6 \mu \mathrm{M})$
C: Antimycin A $(10 \mu \mathrm{M})$
D: PMA (100 $\mathrm{ng} / \mathrm{ml})$

Figure 3. Layout of leukocytes and platelets on XF24 and XF96 analyzer plates. A) Leukocytes (125-250k cells/well) and platelets (25 X $10^{6}$ cells/well) from a single donor are plated on the XF24 assay plate among background controls. $75 \mu$ of $10 x$ stocks of oligomycin ( $0.5 \mu \mathrm{g} /$ $\mathrm{ml})$, FCCP $(0.6 \mu \mathrm{M})$, antimycin-A $(10 \mu \mathrm{M})$, and PMA $(100 \mathrm{ng} / \mathrm{ml})$ diluted in XF media are loaded into the indicated injection ports. B) Leukocytes $\left(75-150 \mathrm{k}\right.$ cells/well) and platelets $\left(10 \times 10^{6} \mathrm{cells} /\right.$ well) from as many as four donors can be plated on the XF96 in a total volume of $180 \mu \mathrm{I} X F-$ DMEM. $20 \mu \mathrm{l}$ of $10 \mathrm{x}$ stocks of oligomycin $(1.0 \mu \mathrm{g} / \mathrm{ml})$, FCCP $(0.6 \mu \mathrm{M})$, antimycin-A $(10 \mu \mathrm{M})$, and PMA $(100 \mathrm{ng} / \mathrm{ml})$ diluted in XF-DMEM are loaded into the indicated injection ports. 


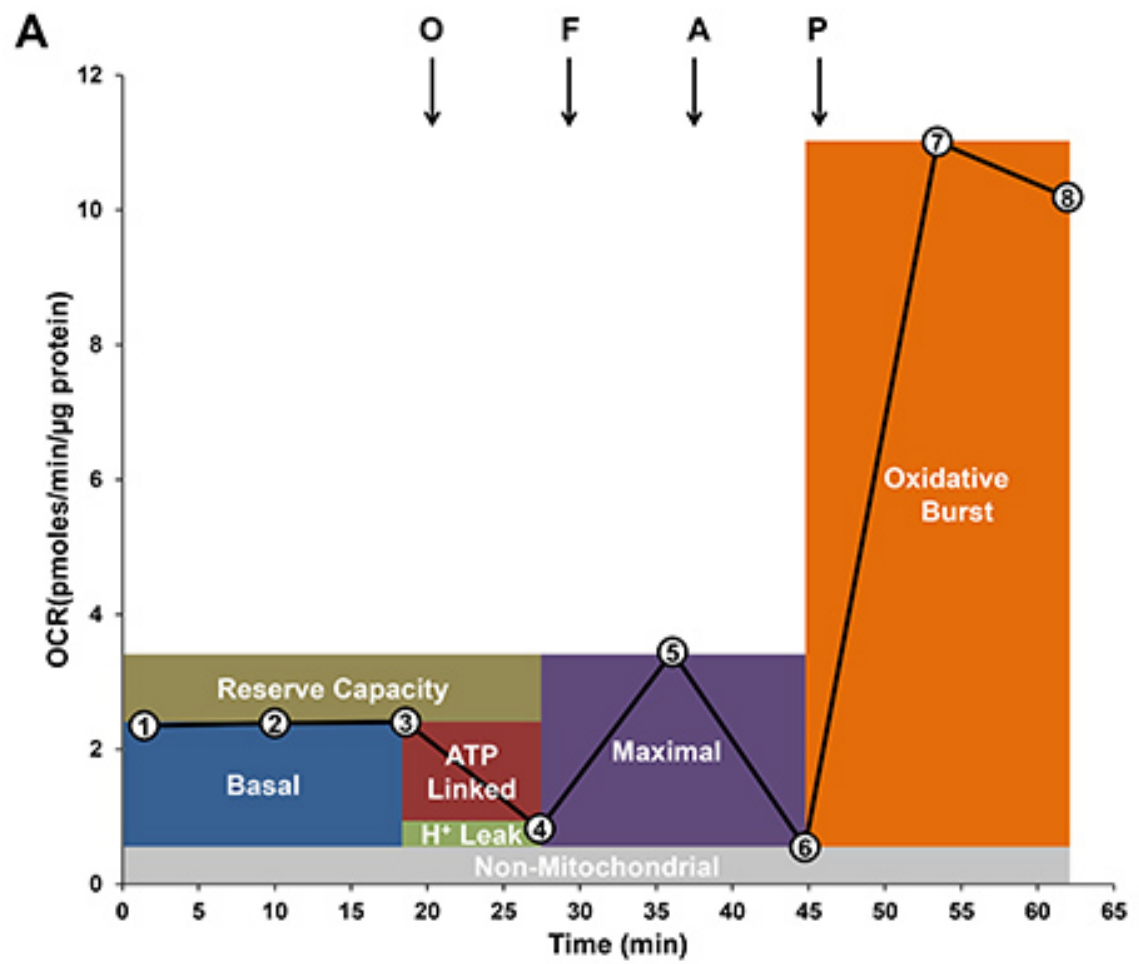

B

$$
\begin{aligned}
\text { Basal } & =(\text { Rate } 3-\text { Rate } 6) \\
\text { ATP-Linked } & =(\text { Rate } 3-\text { Rate } 4) \\
\text { Proton }\left(\mathrm{H}^{+}\right) \text {Leak } & =(\text { Rate } 4-\text { Rate } 6) \\
\text { Maximal } & =(\text { Rate } 5-\text { Rate } 6) \\
\text { Reserve Capacity } & =(\text { Rate } 5-\text { Rate } 3) \\
\text { Oxidative Burst } & =(\text { Rate } 7-\text { Rate } 6)
\end{aligned}
$$

Figure 4. Analysis of Bioenergetic indices. A) Representative trace of oxygen consumption rate (OCR) by mitochondria and oxidative burst in monocytes. Basal OCR is established (first 3 rates indicated by numbers in circles). Then sequential additions of oligomycin (O; $0.5 \mu \mathrm{g} / \mathrm{ml})$, FCCP $(F ; 0.6 \mu \mathrm{M})$ and antimycin-A $(A ; 10 \mu \mathrm{M})$ are injected to determine mitochondrial and nonmitochondrial respiration of nonactivated cells. Finally, PMA $(100 \mathrm{ng} / \mathrm{ml})$ is added to measure oxidative burst. B) Modular analysis of mitochondrial function and oxidative burst are calculated by the differences in the rates as indicated.

\begin{tabular}{|l|l|l|l|l|}
\hline & Optimal Seeding Density & $\begin{array}{l}\text { Avg. Basal OCR }\left(\mathrm{pmol} \mathrm{O}_{2} /\right. \\
\mathrm{min})\end{array}$ & $\begin{array}{l}\text { Avg. Oxidative Burst OCR } \\
\left(\mathrm{pmol} \mathrm{O}_{2} / \mathrm{min}\right)\end{array}$ & Avg. protein $(\mu \mathrm{g}) /$ well \\
\hline Monocytes & 250k cells / well & $83.9( \pm 13.0)$ & $300.3( \pm 58.8)$ & $19.0( \pm 2.4)$ \\
\hline Neutrophils & $250 \mathrm{k}$ cells / well & $6.1( \pm 2.2)$ & $1411.8( \pm 233.3)$ & $20.6( \pm 2.7)$ \\
\hline Lymphocytes & $250 \mathrm{k}$ cells / well & $52.9( \pm 7.7)$ & $15( \pm 4.1)$ & $13.2( \pm 2.1)$ \\
\hline Platelets & $25 \times 10^{6}$ cells / well & $199.4( \pm 20.3)$ & $24( \pm 2.7)$ & $47.5( \pm 3.1)$ \\
\hline
\end{tabular}

Table 1. Normal parameters for the XF24 assay: The average basal (Rate 3) and oxidative burst (Rate 7) OCR not corrected to nonmitochondrial OCR or protein are shown by cell type plated at the indicated cell densities. The average protein content per well is shown as performed by a DC Lowry assay. These data represent the mean values of 6-8 healthy donors with parenthesis containing \pm SEM.

\begin{tabular}{|l|l|l|l|}
\hline Step & Problem & Possible reason & Solution \\
\hline
\end{tabular}




\begin{tabular}{|c|c|c|c|}
\hline 1.2 & clear plasma & $\begin{array}{l}\text { platelets pelleted during } \\
\text { centrifugation }\end{array}$ & $\begin{array}{l}\text { decrease centrifugation time to } 10 \\
\text { min or slow to } 400 \times g\end{array}$ \\
\hline & milky plasma & excess lipids & avoid postprandial collection \\
\hline \multirow[t]{2}{*}{1.6} & incomplete cell bands formed & $\begin{array}{l}\text { improper gradient preparation, cold } \\
\text { reagent }\end{array}$ & $\begin{array}{l}\text { avoid disrupting gradient during } \\
\text { pipetting, use room temperature } \\
\text { reagent }\end{array}$ \\
\hline & clumps in cell bands & $\begin{array}{l}\text { clotting of blood may have } \\
\text { occurred }\end{array}$ & $\begin{array}{l}\text { Collect blood using anticoagulants } \\
\text { such as ACD or EDTA }\end{array}$ \\
\hline \multirow[t]{2}{*}{1.10} & no cell pellet & see step 1.6 & If supernatant hazy see below \\
\hline & hazy supernatant & $\begin{array}{l}\text { Heavy Ficoll gradient } \\
\text { contamination, platelet } \\
\text { contamination }\end{array}$ & $\begin{array}{l}\text { Increase centrifugation speed to } \\
900 \times \text { g, dilute with more RPMI }\end{array}$ \\
\hline $1.16,1.17$ & low yield of leukocytes & $\begin{array}{l}\text { heavy RBC contamination, not } \\
\text { enough whole blood, clotting }\end{array}$ & $\begin{array}{l}\text { Double antibody and RPMI-BSA } \\
\text { volumes, collect more blood, add } \\
\text { anticoagulant }\end{array}$ \\
\hline 1.19 & platelet aggregation & $\begin{array}{l}\mathrm{PGI}_{2} \text { omitted, exposure to cold } \\
\text { media, prolonged storage in } \\
\text { plasma }\end{array}$ & $\begin{array}{l}\text { add } \mathrm{PGI}_{2} \text { to platelet wash buffer, } \\
\text { use room temperature reagents }\end{array}$ \\
\hline 1.20 & low platelet count & $\begin{array}{l}\text { loss during primary centrifugation, } \\
\text { platelet aggregation }\end{array}$ & See steps 1.2 and 1.19 \\
\hline 2.3 & RBC contamination & & Repeat MACS separation \\
\hline
\end{tabular}

Table 2. Leukocyte and platelet isolation troubleshooting. The table lists common problems encountered during leukocyte and platelet cell isolation from whole blood and solutions that may guide users through the troubleshooting process.

\section{Discussion}

This protocol represents the compilation of several commonly utilized techniques for blood cell isolation in a manner suitable for bioenergetics analysis. The contiguous techniques presented are advantageous to other isolation methods (i.e. FACs analysis) for their ability to isolate large numbers of cells in controlled media conditions with minimal stresses placed on the isolated cells. It has the disadvantage of lengthy isolations even with minimal interruptions. This protocol serves as the basis for the isolation of primary blood cells from human subjects, which can be extrapolated into clinical settings and translational research.

MACS separation is a reliable cell isolation technique that offers the possibility of isolating cells directly from whole blood; however, this method requires greater amounts of antibody and is not optimal for the isolation of all four distinct cell types as described in this method. There has been no evidence to show that the positive selection of leukocytes by MACS separation results in activation using our isolation protocol. MACS columns function by sequestration of labeled cells in a magnetic field using antibodies conjugated to $50 \mathrm{~nm}$ superparamagnetic particles. Labeled cells are then eluted off the column. Positive and negative selections are implemented in this protocol to ensure rapid isolation and purity. If inadequate cell numbers are obtained from isolation or purity is in question, more antibodies can be added to the sample as per the vendor's instruction and second passage through the LS column may result in greater purity (Table 2). Our laboratory found high purity and plating efficiency using the existing protocol by analyzing final cell suspensions by FACS analysis ${ }^{8}$.

Extracellular flux analyzers have the ability to monitor both oxygen consumption and media acidification in real time over that of other electrodes. The oxygen consumption as observed by the oxidative burst response in monocytes and neutrophils is NADPH oxidase dependent as shown by inhibition with $\mathrm{DPI}^{8}$. We have seen a time dependent loss in oxidative burst capacity with prolonged isolations or extended XF assays. This protocol was designed and developed for use on the XF24 but is also compatible with the XF96 at approximately a third to one half of the XF24 cell seeding densities (Figure 3).

In design of this protocol, adherence to existing protocols for each technique was required for optimal performance with modifications made solely to control media conditions for bioenergetic analysis. After mastering techniques, such a protocol can be used in a wide array of translational and research applications to measure the toxicity or effectiveness of treatment strategies, explore metabolic characteristics of disease, and oxidant production by monocyte and neutrophils in inflammatory conditions.

\section{Disclosures}

VDU is a member of the Seahorse Bioscience Scientific Advisory Board.

\section{Acknowledgements}

The authors would like to acknowledge the technical contribution of Gloria A Benavides. This work was supported by American Heart Association 13PRE16390001 (SR), NIH T32HL07918 (PAK), NIH T32HL007457 (TM), P30DK056336 (BKC), NIDDK Diabetic Complications Consortium (DiaComp, www.diacomp.org) grant DK076169 (subaward VDU), and the O'Brien Center P30 DK079337 (VDU). 


\section{References}

1. Mancuso, M. et al. Mitochondria, cognitive impairment, and Alzheimer's disease. Int. J. Alzheimer's Dis. 2009, doi:10.4061/2009/951548 (2009).

2. Jin, S. Autophagy, mitochondrial quality control, and oncogenesis. Autophagy. 2, 80-84 (2006).

3. Cordero, M. D. et al. Mitochondrial dysfunction and mitophagy activation in blood mononuclear cells of fibromyalgia patients: implications in the pathogenesis of the disease. Arthritis Res. Ther. 12, R17, doi:10.1186/ar2918 (2010).

4. Japiassu, A. M. et al. Bioenergetic failure of human peripheral blood monocytes in patients with septic shock is mediated by reduced F1Fo adenosine-5'-triphosphate synthase activity. Crit. Care Med. 39, 1056-1063, doi:10.1097/CCM.0b013e31820eda5c (2011).

5. Widlansky, M. E. et al. Altered mitochondrial membrane potential, mass, and morphology in the mononuclear cells of humans with type 2 diabetes. Transl. Res. 156, 15-25, doi:10.1016/j.trsl.2010.04.001 (2010).

6. Shi, C. et al. Effects of ageing and Alzheimer's disease on mitochondrial function of human platelets. Exp. Gerontol. 43, 589-594, doi:10.1016/j.exger.2008.02.004 (2008).

7. Avila, C. et al. Platelet mitochondrial dysfunction is evident in type 2 diabetes in association with modifications of mitochondrial anti-oxidant stress proteins. Exp. Clin. Endocrinol. Diabetes. 120, 248-251, doi:10.1055/s-0031-1285833 (2012).

8. Chacko, B. K. et al. Methods for defining distinct bioenergetic profiles in platelets, lymphocytes, monocytes, and neutrophils, and the oxidative burst from human blood. Lab. Invest. 93, 690-700, doi:10.1038/labinvest.2013.53 (2013).

9. Hill, B. G. et al. Integration of cellular bioenergetics with mitochondrial quality control and autophagy. Biol. Chem. 393, 1485-1512, doi:10.1515/hsz-2012-0198 (2012).

10. Hultqvist, M. et al. Enhanced autoimmunity, arthritis, and encephalomyelitis in mice with a reduced oxidative burst due to a mutation in the Ncf1 gene. Proc. Natl. Acad. Sci. U.S.A. 101, 12646-12651, doi:10.1073/pnas.0403831101 (2004).

11. Rosa-Borges, A. et al. Glucose-6-phosphate dehydrogenase deficiency with recurrent infections: case report. J. Ped. 77, 331-336 (2001).

12. Walkowiak, B., Kesy, A. \& Michalec, L. Microplate reader--a convenient tool in studies of blood coagulation. Thromb. Res. 87, 95-103 (1997).

13. Dranka, B. P. et al. Assessing bioenergetic function in response to oxidative stress by metabolic profiling. Free Radic. Biol. Med. 51, 1621-1635, doi:10.1016/j.freeradbiomed.2011.08.005 (2011).

14. Nicholls, D. G. et al. Bioenergetic profile experiment using C2C12 myoblast cells. J. Vis. Exp. (46), e2511, doi:10.3791/2511 (2010). 